The Psychological Record, 2009, 59, 347-370

\title{
EXTINCTION-INDUCED VARIABILITY IN HUMAN BEHAVIOR
}

\author{
Jennifer M. Kinloch, T. Mary Foster, and James S. A. McEwan \\ University of Waikato
}

\begin{abstract}
Participants earned points by pressing a computer space bar (Experiment 1) or forming rectangles on the screen with the mouse (Experiment 2) under differential-reinforcement-of-low-rate schedules, followed by extinction. Variability in interresponse time (the contingent dimension) increased during extinction, as for Morgan and Lee (1996); variability in diagonal length (the noncontingent dimension, Experiment 2) did not. In Experiment 3, points were contingent on rectangle size. Rectangle size and interresponse-time (the noncontingent dimension) variability increased in extinction. There was greater variability in the contingent dimension during extinction for participants with the more varied history of reinforcement in Experiment 2 but not in Experiment 3. Overall, variability in the contingent dimension increased in extinction, but the degree of increase was affected by reinforcement history.
\end{abstract}

Neuringer (2002) argued that variability in behavior is functional, adaptive, and essential to the development of novel, complex, and efficient behavior (Dewitte \& Verguts, 1999; Neuringer, Deiss, \& Olsen, 2000). Neuringer asserted that variability can be changed by reinforcement and be a product of extinction. Under extinction, responses that led to reinforcers in the past are made ineffective. This often results in increases in response rate and increased variation in various dimensions of the behavior, including magnitude and topography (Neuringer, 2002). Neuringer (2004) reported extensive research on the reinforcement of variability, but while extinction-induced variability appears essential for the development of behaviors, little research has been conducted in this area.

Research on extinction-induced variability has typically compared performance on reinforcement schedules with performance during extinction. Animal studies have found greater variability during extinction than during reinforcement (Antonitis, 1951; Eckerman \& Lanson, 1969; Lachter \& Corey, 1982; Neuringer, Kornell, \& Olufs, 2001; Stokes, 1995), with variability increasing as a function of reinforcer intermittency (Eckerman \& Lanson, 1969; Lachter \& Corey, 1982). Herrnstein (1961), however, found greater variability during continuous reinforcement (CRF) than during intermittent schedules, but his results remain unreplicated. Antonitis (1951) and Eckerman and Lanson (1969) measured variability in extinction of response location, but

Correspondence concerning this article should be addressed to Jennifer Kinloch, Department of Psychology, University of Waikato, Private Bag 3105, Hamilton, New Zealand. E-mail: jmk11@ waikato.ac.nz. 
reinforcement had not been contingent on location. Similarly, Stokes (1995) measured variability in the form of the bar press, where reinforcement was contingent simply on bar depression. In these studies, the variability studied was in a noncontingent response dimension. Lachter and Corey (1982) and Neuringer et al. (2001) measured variability in extinction of a dimension of the response on which reinforcement was contingent (response duration and sequence variability, respectively). In summary, animal studies show that the variability of the contingent and noncontingent dimensions of responses may increase in extinction.

There are only two studies that have examined the effects of extinction on response variability with humans (Maes, 2003; Morgan \& Lee, 1996). In the Maes study, based on Neuringer et al. (2001), responses were made across three computer keys, and when a sequence of presses met the variability criterion, there was either no consequence (the extinction condition) or the word "correct" was presented on the computer screen (the feedback condition). Removal of feedback resulted in decreases in sequence variability, contrary to Neuringer et al.'s findings.

Maes's (2003) data did not support the idea that extinction results in increased variability in response sequences (the contingent dimension) with humans. Maes suggested that the difference between the studies may be attributable to rats' tendencies to reproduce a reinforced response sequence immediately after a reinforcer. He suggested that when reinforcers were removed the sequence repetition disappeared, thus resulting in increases in sequence variability. The humans did not show this tendency to repeat reinforced sequences and thus extinction could not reduce this repetition.

Morgan and Lee (1996), working with human participants, examined extinction-induced variability in interresponse times (IRTs) following differential-reinforcement-of-low-rate-responding (DRL) schedules. IRT was the contingent dimension in this study. The participants completed a series of DRL schedules in which they were required to press the space bar at less than a defined rate in order to gain points. These schedules were followed by extinction. Morgan and Lee reported that IRTs were more variable during extinction than during the DRL schedules. During the extinction period, IRTs frequently were much longer than those required by the longest of the DRL schedules, as well as frequently being much shorter than those required by the shortest of the DRL schedules. In their second experiment, another set of participants were exposed to one DRL schedule only, followed by extinction. Again, participants exhibited greater IRT variability during extinction than during the DRL schedule. These data suggest that extinction increases variability in this contingent dimension (IRT) with humans.

Maes (2003) and Morgan and Lee (1996) are the only two experimental studies of extinction-induced behavioral variability in humans. Thus, although such variability is thought to be essential for the development of new forms of behavior, there has been little research in this area with humans. Maes and Morgan and Lee both studied variability in a contingent dimension of the reinforced response and found contrary results. There are no studies with humans in which the variability of a dimension of a response not related to the earlier reinforcement has been studied in extinction. Maes reinforced variability in response sequences and then examined this same variability in extinction, presenting a potential confound. Morgan and Lee, on the other hand, used DRL schedules prior to extinction. The response dimension they 
studied in extinction, IRT, was the subject of the previous contingency, but variability in this dimension had not been specifically reinforced. This latter procedure, therefore, seems to provide a clearer picture of the effect of extinction on response variability with humans.

Overall, previous animal research shows that behavioral variability increases during extinction following reinforcement, regardless of whether or not the dimension of behavior being measured had previously been subject to the reinforcement contingency. Morgan and Lee (1996) found similar results with humans, whereas Maes (2003) did not. Most studies have examined only one dimension of the response, and there has been no systematic comparison of the effects of extinction on variability in both contingent and noncontingent dimensions of a behavior within a single study with humans or animals.

Given the importance of extinction in the development of new behavior, one aim of this present study was to explore extinction-induced variability in human behavior further, in particular, to compare effects of extinction on the contingent and noncontingent dimensions of a response. Given the contrary results with humans, the goal of the first experiment was to replicate Morgan and Lee's (1996) procedure to see if their findings could be reproduced.

\section{Experiment 1}

\section{Participants}

Eleven adult participants (P1.1-P1.11) were recruited through notices in the Department of Psychology. Ethical approval was received from the department ethics committee for this and the later experiments in the study. Participants who were enrolled in a first-year (100-level) psychology course received $1 \%$ course credit for each day that they participated, up to a maximum of $4 \%$.

\section{Apparatus}

Experimental events were controlled and responses recorded by a computer program written in Visual Studio. Participants used a Dell 260 personal computer with a 17-in. CRT monitor.

\section{Procedure}

Participation involved a maximum of sixteen 10-min sessions, with a maximum of five sessions in a day. Participants were seated alone at a computer in a room free from distraction within the university. The following instructions, used by Morgan and Lee (1996), were presented on the computer screen:

Thank you for agreeing to participate in this study. Your task is to obtain as many points as possible per session by pressing the space bar. Respond as often as you like. When the session finishes, please leave the room and notify the experimenter. Remember, try to get as many points as you can in each session. When you are ready to begin, press the space bar.

The instructions remained on the screen until the participant pressed the space bar, after which a points counter appeared at the bottom center of the 
screen. The points counter remained on for the rest of the session. With each response that met the current reinforcement criterion, there was a 2-s tone and the points count increased by five.

The procedure was a direct replication of Morgan and Lee (1996). There were two phases, each with a different group of participants. Each participant in Phase 1 responded on three ascending DRL conditions, with one reversal to a previous condition, followed by an extinction session. Participants in Phase 2 responded on one DRL schedule and then an extinction session. The DRL schedules determined the minimum IRT required for a response to gain points. In order to progress to the next schedule or to extinction, a participant's responses were required to meet a stability criterion of less than or equal to two responses per reinforcer (on average) across the last $25 \%$ of obtained reinforcers in that session. If this criterion was not met, the participant was reexposed to that schedule in the next experimental session. Extinction was similar to a DRL session, but responses on the space bar no longer gave points. Extinction lasted for one 10-min session.

The schedules and schedule order for the participants in this and the subsequent experiments are presented in Table 1.

\section{Results}

\section{Phase 1}

All participants in Phase 1 met the stability criterion in the first session for each DRL schedule. Figure 1 shows consecutive IRTs during the session with each DRL schedule and the extinction session for each participant, together with the means and standard deviations for each condition. In this figure and all subsequent figures, the horizontal dashed line on the graphs for the DRL sessions gives the length of IRT required during that session. IRTs that fall above this line resulted in reinforcement. The vertical dotted line shows the beginning of the fourth quarter (Q4), defined by Morgan and Lee (1996) as the part of the final DRL session during which the last 25\% of reinforcers were obtained. To show the data clearly the axes have different scales depending on the number of responses and the degree of variation in IRT. The difference in the number of responses recorded in each schedule is partially due to the fact that fewer responses can be made in $600 \mathrm{~s}$ with longer DRL criteria.

Once a DRL criterion had been met for the first time, the participants in Phase 1 continued to make responses with IRTs that fell at or near the criterion, with very little variability, for the remainder of the session. During the final DRL schedule, the IRTs for 4 of the participants met the DRL criterion after very few responses. All participants had greater maximum IRTs during extinction than during any DRL schedule, exceeding $200 \mathrm{~s}$ for 2 participants. Additionally, all had greater IRT standard deviations and smaller minimum IRTs during extinction than during Q4.

\section{Phase 2}

P1.6 and P1.7 reached the stability criterion in their fourth and fifth sessions, respectively, whereas $\mathrm{P} 1.9$ and $\mathrm{P} 1.11$ reached the stability criterion during their second sessions. The other 2 participants reached the criterion in one session. 


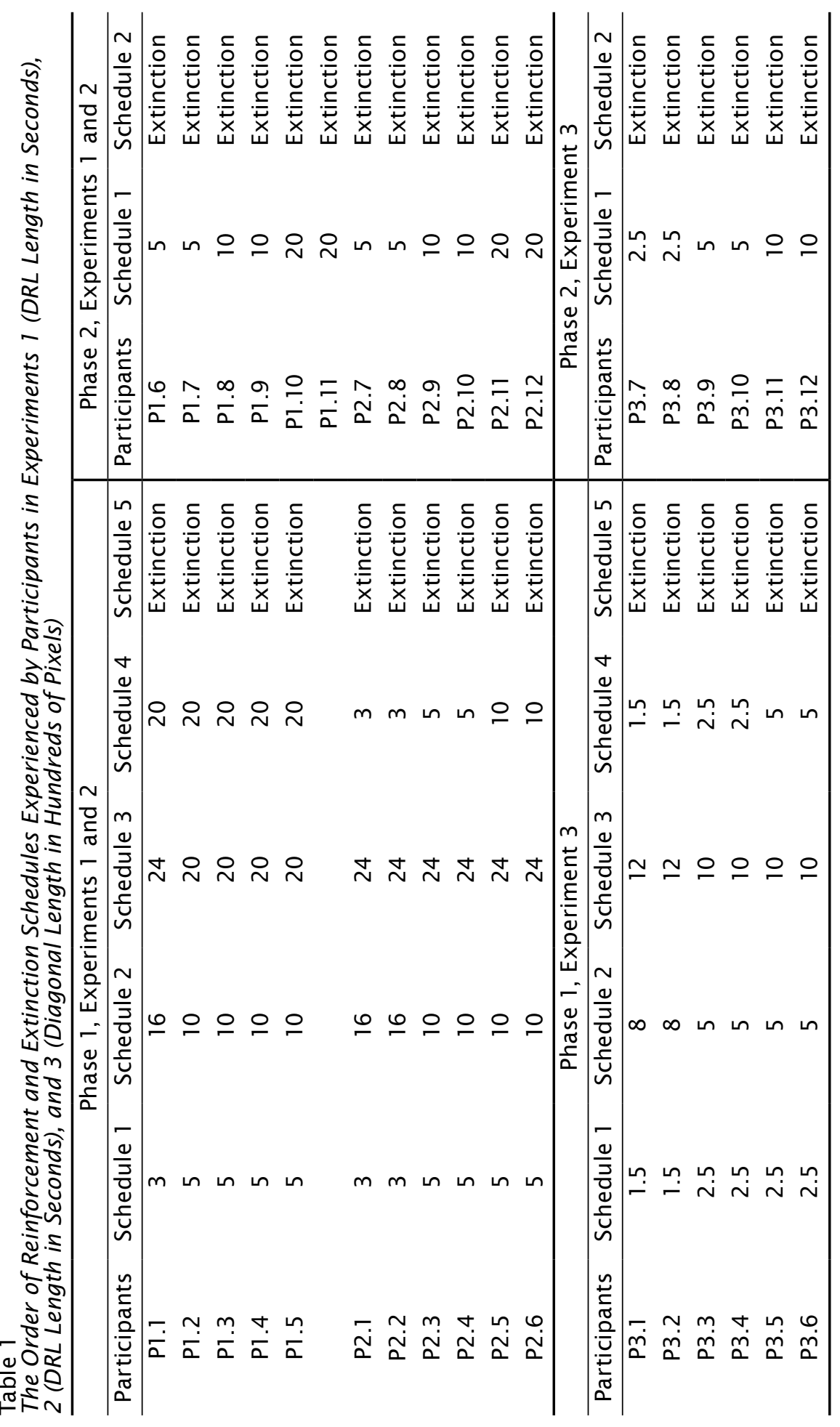



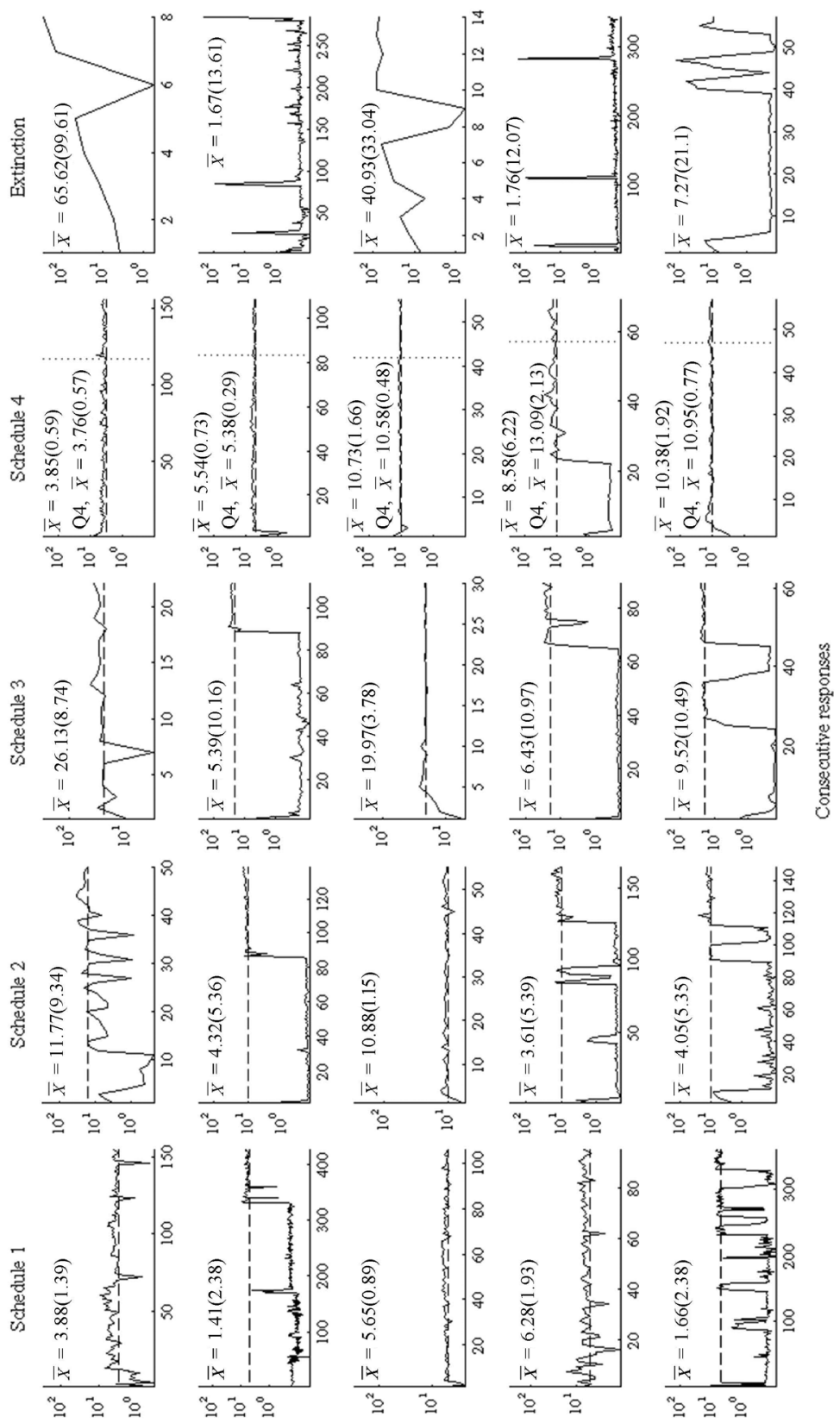

$\overrightarrow{\bar{s}}$

$\frac{m}{a}$

$\stackrel{+}{\stackrel{\Delta}{\Delta}}$

空

(s) $L$ सI soI

Figure 1. Consecutive IRTs during each DRL schedule and extinction for each participant in Phase 1 of Experiment 1. IRT means $(\bar{X})$ are given on each graph, with the standard deviations $(S D)$ given in parentheses. For Schedule 4, both whole session and Q4 means and standard deviations are shown. 
Figure 2 shows the data from the final session with the DRL schedule and extinction. Several participants made a large number of unreinforced responses prior to meeting the DRL criterion. Once the IRTs for these participants met the criterion, subsequent IRTs were close to the DRL criterion for the remainder of the session. Q4 contains very few responses for the participants who made a large number of unreinforced responses before meeting the DRL criterion. IRT was more variable during extinction than during any of the DRL schedules for 5 participants. All Phase 2 participants had larger IRT standard deviations, greater maximum IRTs, and smaller minimum IRTs during extinction than during Q4.
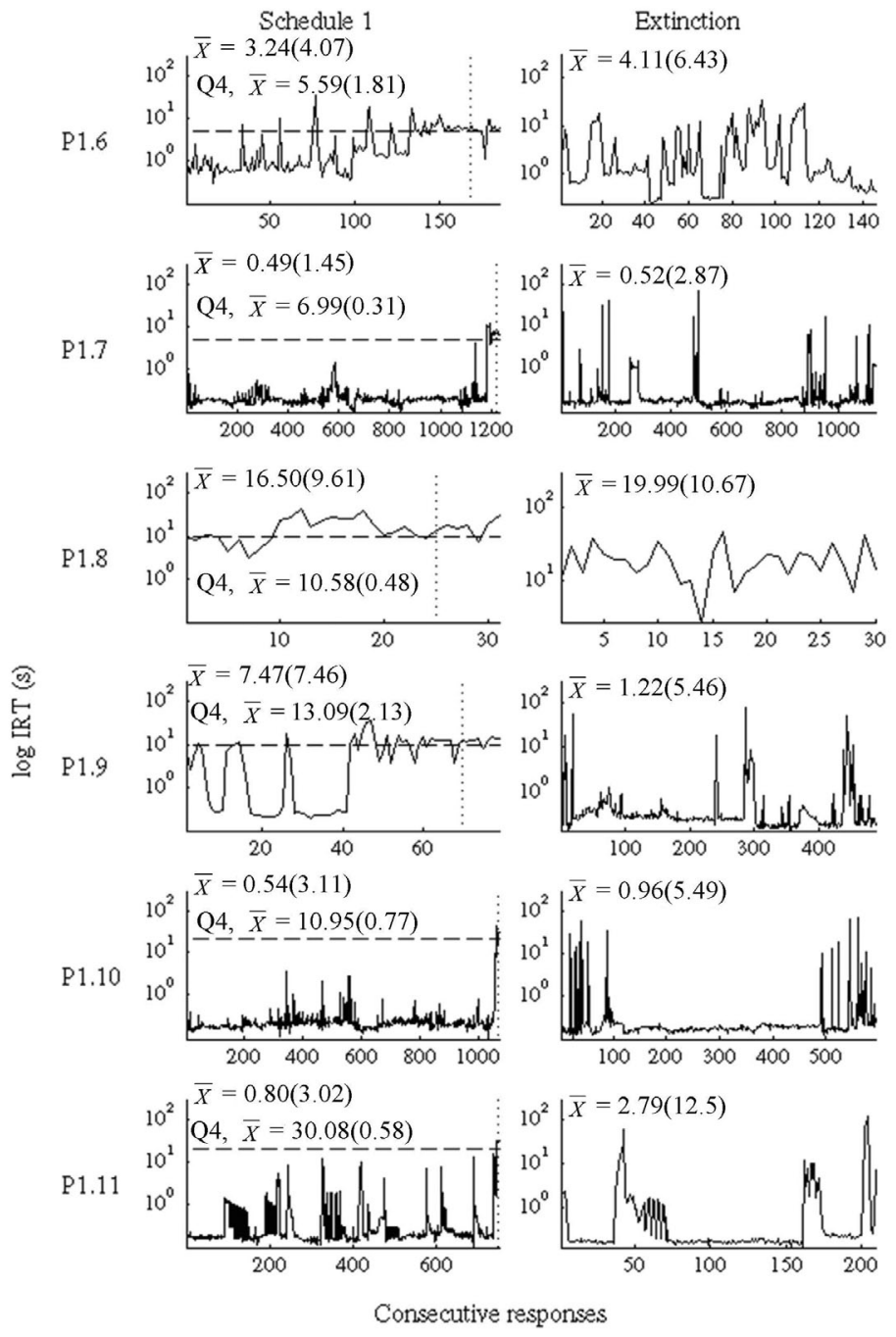

Figure 2. Consecutive IRTs during the DRL schedule and extinction for each participant in Phase 2 of Experiment 1. Whole session and Q4 IRT means $(\bar{X})$ are given on each graph, with the standard deviations $(S D)$ given in parentheses. 


\section{Discussion}

All participants exhibited greater IRT variability (assessed from the standard deviations) during extinction than during Q4, a finding similar to Morgan and Lee (1996). The observed variability during Q4 was similar to Morgan and Lee in that the IRTs in Q4 fell at or near the DRL criterion for that session. It is not possible to compare the actual values to those in the Morgan and Lee study, as they did not provide that data. The present study extends Morgan and Lee's findings by showing that IRT standard deviations were greater during extinction than during the final sessions of any of the preceding DRL schedules.

These data failed to replicate the finding of Morgan and Lee (1996) that reinforcement history has no effect on IRT variability during extinction. In both studies the participants in Phase 1 had a history of reinforcement for a range of IRTs, whereas those in Phase 2 had experienced reinforcers for only one. Whereas Morgan and Lee reported similar variability in extinction for Phase 1 and Phase 2, the current study found that Phase 1 resulted in greater IRT variability during extinction than Phase 2. Comparisons of the data with Morgan and Lee's suggest that the differences lie in Phase 1 rather than Phase 2, with much higher maximum IRTs recorded during extinction in Phase 1 of this study than in either phase of Morgan and Lee's study.

Not only did the number of DRL schedules experienced differ between the phases in this study, but the proportion of responses that resulted in reinforcers also differed. The participants in Phase 2 made many more unreinforced responses before reaching stability than did those in Phase 1 . Three of the participants in Phase 2 made an exceptionally low number of reinforced responses, with only $1 \%$ to $3 \%$ of their responses resulting in a reinforcer. This may help account for the differences in variability during extinction for Phases 1 and 2, as extinction might not have been discriminable from the DRL schedule preceding it for some Phase 2 participants. Morgan and Lee (1996) do not provide enough detailed data to see if such effects were present in their findings. The present data suggest then that, contrary to Morgan and Lee, the number of DRL schedules experienced and/or the proportion of unreinforced responses had an effect on extinction-induced IRT variability in humans.

Extinction is said to affect more than the reinforced, or contingent, dimension of a response. Variability in noncontingent aspects of the response (such as its form) is part of the process of developing new forms of responses. In the present experiment, IRT was the contingent dimension of the response. To study the effects of extinction on a noncontingent dimension, another aspect of the response must be measured. The response of operating a space bar on the computer keyboard is not one that allows easy measurement of variations in form. While it is simple to measure IRT, and it would also be possible to measure duration, IRT and duration are confounded. To study extinction-induced variability further requires a response with measurable dimensions that could vary independently of IRT.

The task selected for the next experiment was based on a study by Ross and Neuringer (2002) in which participants drew rectangles on the screen with the computer mouse. Ross and Neuringer measured response variability in terms of shape, area, and location of the rectangle on the screen.

In the next experiment, reinforcers were arranged under the same DRL 
schedules as in Experiment 1. However, participants were asked to produce rectangles on the screen. IRT and rectangle location on the screen were recorded. The goal was to see if extinction has an effect on the variability of a noncontingent dimension of a response and to examine further the differences observed between the present study and Morgan and Lee (1996).

\section{Experiment 2}

\section{Participants}

The 12 adult participants (P2.1-P2.12) were recruited and course credit was allocated as in Experiment 1.

\section{Apparatus}

The computer program used in Experiment 1 was modified so that the required response was drawing a rectangle on the screen using the mouse. All other apparatus were the same as in Experiment 1.

\section{Procedure}

The procedure was similar to Experiment 1 except that the participants were required to draw rectangles on the screen. To draw a rectangle the participant was required to depress the left mouse button when the cursor was at the desired starting point and, while holding the button down, move the cursor to the location of the opposing corner of the rectangle. Releasing the left mouse button determined the end location of the rectangle. Thus, the instructions for Experiment 2 differed from those of Experiment 1 only in terms of the topography of the response required:

Thank you for agreeing to participate in this study. Your task is to obtain as many points as possible per session by drawing rectangles on the screen using the mouse. Respond as often as you like. When the session finishes, please leave the room and notify the experimenter. Remember, try to get as many points as you can each session. When you are ready to begin, click the left mouse button.

Once a participant clicked the left mouse button, the instructions disappeared and a points counter appeared in the bottom center of the screen. As in Experiment 1, each correct response resulted in a 2-s tone and an increase of 5 points. The order of DRL schedules is given in Table 1. Recording the start and end locations provided the parameters of the rectangle (including diagonal length).

\section{Results}

\section{Phase 1}

P2.1 required two sessions on the second DRL schedule, and P2.4 and P2.5 required two sessions on their first DRL schedules to reach stability. In all other cases stability was achieved in one session. 
IRT. Figure 3 shows consecutive IRTs during the last session of each DRL schedule and during extinction, together with means and standard deviations, for each participant. Nearly all IRTs were at or near the DRL criterion throughout the final DRL session and during Q4. IRTs were more variable during extinction than in any of the DRL schedules or during Q4 for all participants. Maximum IRTs for all participants in the extinction session were greater than those in any of the DRL schedules. The minimum IRTs during extinction were smaller than those during Q4. All participants in Phase 1 had larger IRT standard deviations during extinction than during Q4.
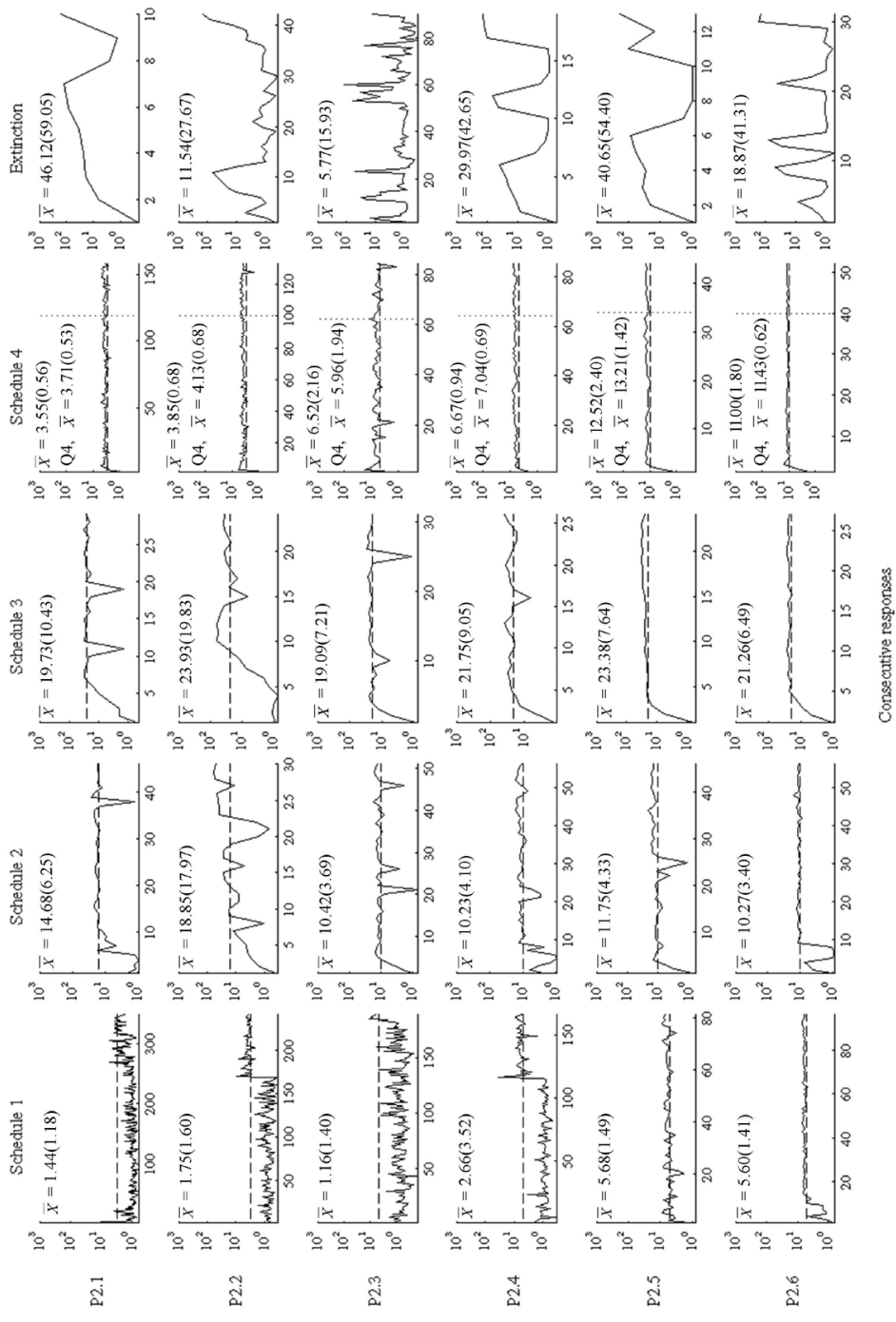

(s) $\mathrm{L}$ प्I $\mathrm{BO}$

Figure 3. Consecutive IRTs during each DRL schedule and extinction for each participant in Phase 1 of Experiment 2. IRT means $(\bar{X})$ are given on each graph, with the standard deviations (SD) given in parentheses. For Schedule 4, both whole session and Q4 means and standard deviations are shown. 
Diagonal length. Figure 4 shows the diagonal length of consecutive responses during the last session with each DRL schedule and during extinction (including the means and standard deviations). Generally, diagonal length was variable in the first DRL but variability was much lower for the following DRL sessions. All participants had more variable diagonal lengths (in terms of larger standard deviations and higher maximum values) during extinction than during Q4. P2.1, P2.4, and P2.5 all had smaller minimum diagonal lengths in Q4 than in extinction.
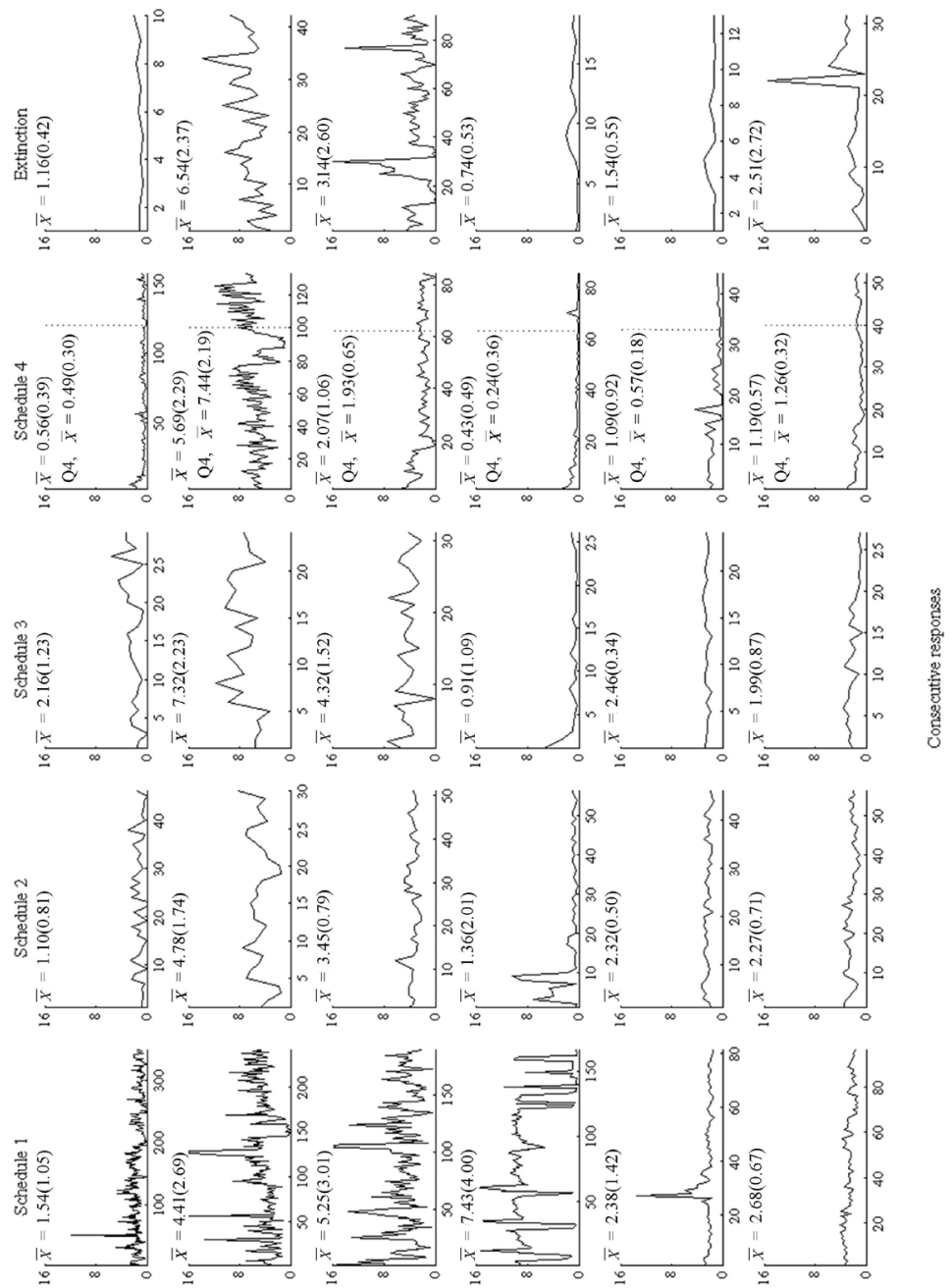

$\overline{\mathrm{j}}$

$\Xi$

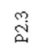

$\stackrel{9}{9}$

בั

¿̊ำ

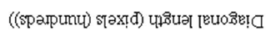

Figure 4. Consecutive diagonal lengths during each DRL schedule and extinction for each participant in Phase 1 of Experiment 2. IRT means $(\bar{X})$ are given on each graph, with the standard deviations $(S D)$ given in parentheses. For Schedule 4, both whole session and Q4 means and standard deviations are shown. 


\section{Phase 2}

P2.7 and P2.12 required one extra session and P2.10 required three extra sessions to reach stability during the DRL schedule; the other 3 participants required one session only.

IRT. Figure 5 shows that IRTs for P2.7 and P2.10 met the criterion near the start of the final session with the DRL, with most IRTs falling close to the criterion once the contingency was met. P2.8 and P2.11 met the criterion after a number of responses, and others did not meet the criterion until the majority of the responses in the session had been made. Five participants in Phase 2 had higher levels of IRT variability during extinction than during the entire last session with the DRL. All participants had higher IRT standard deviations, greater maximum IRTs, and smaller minimum IRTs during extinction than during Q4. However, the differences in standard deviations were not as large as those for participants in Phase 1.
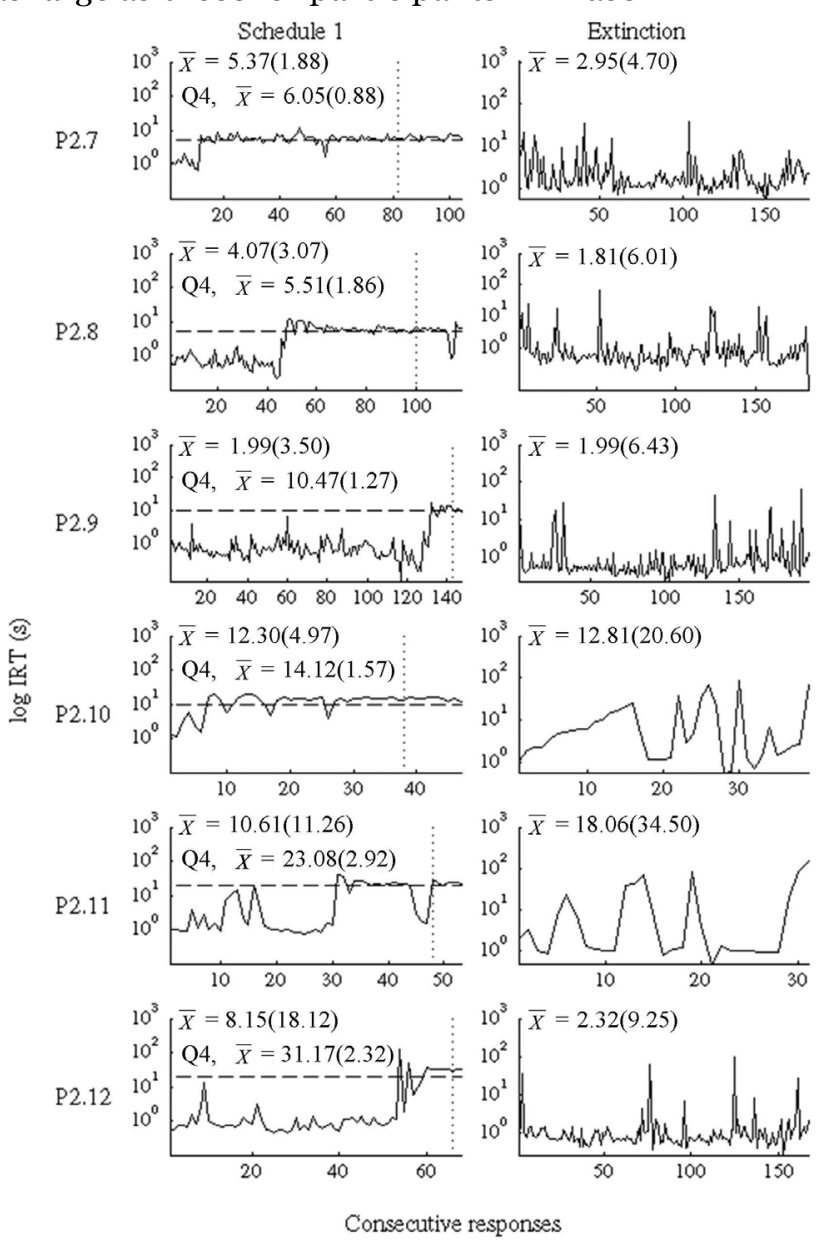

Figure 5. Consecutive IRTs during the DRL schedule and extinction for each participant in Phase 2 of Experiment 2. Whole session and Q4 IRT means $(\bar{X})$ are given on each graph, with the standard deviations $(S D)$ given in parentheses. 
In order to compare variability, both within and across experiments, a repeated-measures ANOVA was performed, using IRT standard deviations from Q4 and extinction and from both Experiments 1 and 2. This found significant differences within subjects, $F(1,19)=31.138, p<0.05, \eta_{p}{ }^{2}=.621$, and between phases, $F(1,19)=10.930, p<0.05, \eta_{p}{ }^{2}=.365$. IRT standard deviations did not differ significantly between Experiments 1 and 2, $F(1$, 19) $=0.505, p>0.05, \eta_{p}{ }^{2}=.024$, nor was there a significant interaction between experiments and phases, $F(1,19)=0.021, p>0.05, \eta_{p}{ }^{2}=.001$. However, there was a significant within-subject interaction between $\mathrm{Q} 4$ and extinction and phase, $F(1,19)=11.36, p<.05, \eta_{p}{ }^{2}=.374$. This is seen as larger increases in standard deviation in extinction for those in Phase 1 than those in Phase 2. No other interactions were significant.

Diagonal length. Figure 6 shows that diagonal length was variable over both the last session of the DRL and extinction. All participants in Phase 2 had greater variability in diagonal length (larger standard deviations, greater maximums, and smaller minimums) during extinction than during Q4. A repeated-measures ANOVA showed diagonal length standard deviations from Q4 and extinction in Phase 1 were not significantly different from those in Phase $2, F(1,10)=4.604, p>0.05, \eta_{p}{ }^{2}=.315$. Within-subject differences in diagonal length standard deviation between Q4 and extinction in Experiment 2 were significant, $F(1,10)=10.481, p<0.05, \eta_{p}{ }^{2}=.534$, but the interaction was not significant, $F(1,10)=1.369, p>0.05, \eta_{p}^{2}=.120$.

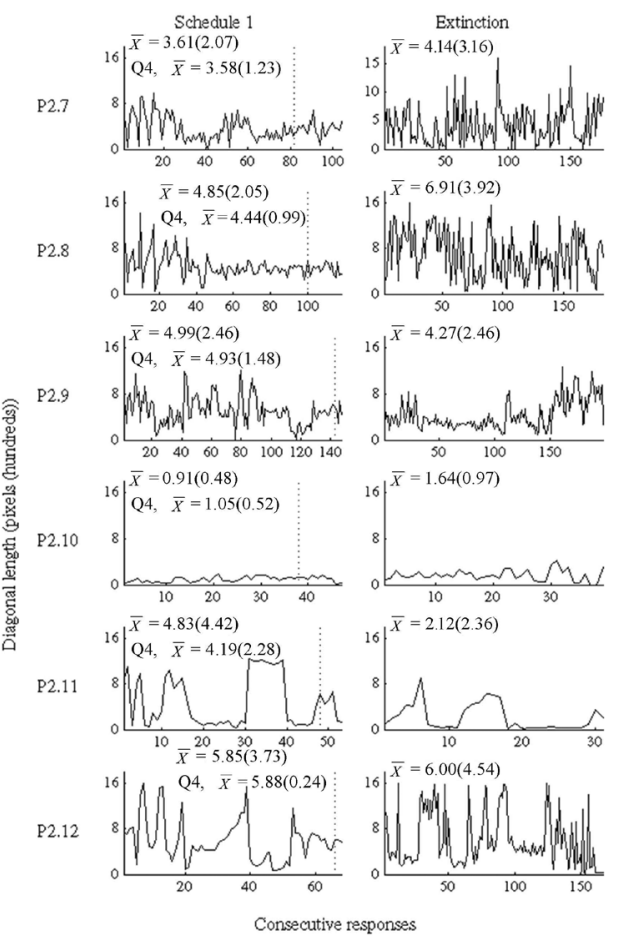

Figure 6. Consecutive diagonal lengths during the DRL schedule and extinction for each participant in Phase 2 of Experiment 2. Whole session and Q4 IRT means $(\bar{X})$ are given on each graph, with the standard deviations $(S D)$ given in parentheses. 


\section{Discussion}

In Experiment 2, IRTs were more variable during extinction than they were during Q4 in both phases. There was also greater variability in IRTs during extinction in Phase 1 than in Phase 2, a finding similar to that in Experiment 1. Thus, both results of Experiment 1 were replicated with this new response.

The length of the diagonal was a noncontingent dimension of the response. For most of the participants, variability in diagonal length decreased across the schedules (Phase 1) and decreased with more exposure to any one DRL (Phase 1 and Phase 2). Diagonal length variability also generally increased in extinction compared to Q4, for both phases. However, contrary to the pattern of IRT variability, diagonal length variability was not significantly different between Phases 1 and 2.

In summary, the findings in Experiment 2 replicated both Experiment 1 and Morgan and Lee (1996), in that variability in the contingent dimension (IRT) was greater during extinction than during reinforcement. They confirm the finding of Experiment 1 that experiencing four DRL schedules gave greater IRT variability in extinction than did experiencing only one DRL schedule. This is contrary to Morgan and Lee's conclusion that reinforcement history does not affect variability in extinction. The present data also extend previous findings by showing that variability in a noncontingent dimension (diagonal length) increased in extinction. However, it appears that reinforcement history had no effect on this noncontingent dimension.

The next experiment kept the response the same but changed the contingent dimension from IRT to diagonal length. The intention was to test the generality of the findings from the first two experiments.

\section{Experiment 3}

\section{Participants}

The 12 adult participants (P3.1-P3.12) were recruited and course credit was allocated as in Experiments 1 and 2.

\section{Apparatus}

The computer program used in Experiment 2 was modified so that reinforcement was contingent on the distance between opposite corners of the rectangle that was drawn.

\section{Procedure}

The procedure, including the instructions and stability criterion, was the same as in Experiment 2. The only difference was that the schedules were changed so that the criterion for gaining the points was based on the length of the diagonal between opposing corners of the rectangle, measured in hundreds of pixels. The diagonal lengths (in hundreds of pixels) required to gain points for P3.1 to P3.12 are shown in Table 1.

The same measures were recorded as in Experiment 2. Also, as in Experiment 2, it should be noted that when comparing the degree of variability across response topographies, diagonal length and response location were constrained by the size of the screen. 


\section{Results}

\section{Phase 1}

All participants in Phase 1 reached the stability criterion in the first session with each schedule.

Diagonal length. Figure 7 shows the diagonal length of consecutive responses for each schedule and extinction. The dashed horizontal line indicates the diagonal length criterion for that session, and the start of Q4 is shown on the graph for the final schedule. There were many more responses in a session than in the previous two experiments. All participants in Phase 1 met the diagonal length criterion near the beginning of the first schedule and diagonal length changed as the criterion changed in each schedule. Some participants frequently produced diagonal lengths greater than the criterion length required to gain reinforcement. Variability of diagonal length was greater in extinction than in Q4 for all participants. Diagonal length standard deviations and the maximum diagonal length values (which were close to the maximum possible due to screen size) were greatest during extinction for all participants. All participants had smaller minimum diagonal length values during extinction than during Q4.

IRT. Figure 8 shows consecutive IRTs for Phase 1. There was very little variability in IRT for all participants during any of the schedules. There was greater variability in IRT during extinction than in Q4 for all of the participants; however, this difference was small for 3 participants.

\section{Phase 2}

All participants in Phase 2 reached the stability criterion in one session.

Diagonal length. Figure 9 shows consecutive diagonal lengths for Phase 2. Five of the 6 participants met the reinforcement criterion near the start of the session. P3.12 met the criterion after approximately 50 responses. Once the criterion had been met, responses continued to be at or near the reinforcement criterion for the remainder of the session. There was greater variability in diagonal length during extinction for all participants. All maximum diagonal lengths were at or near the maximum possible diagonal length (constrained to screen size) during extinction.

A repeated-measures ANOVA showed that the increase in diagonal length standard deviations between Q4 and extinction was statistically significant, $F(1,10)=39.604, p<0.05, \eta_{p}^{2}=.878$, and that diagonal length standard deviations were not significantly different between Phases 1 and $2, F(1,10)=.972, p>0.05, \eta_{p}^{2}=.08$.

IRT. Figure 10 shows that the IRTs for all participants were not very variable when the reinforcement contingency on diagonal length was in effect. IRT standard deviations were less than $1 \mathrm{~s}$, and the means were very similar for all participants when the contingency was operative. Extinction resulted in larger IRT standard deviations and greater maximum IRTs for all of the participants than did the reinforcement schedule or Q4.

A repeated-measures ANOVA showed that the increase in IRT standard deviations from Q4 to extinction was significant, $F(1,10)=5.781, p<0.05$, $\eta_{p}{ }^{2}=.366$, and that there was no significant difference in IRT standard deviations between Phases 1 and 2, $F(1,10)=.473, p>0.05, \eta_{p}{ }^{2}=.045$. 

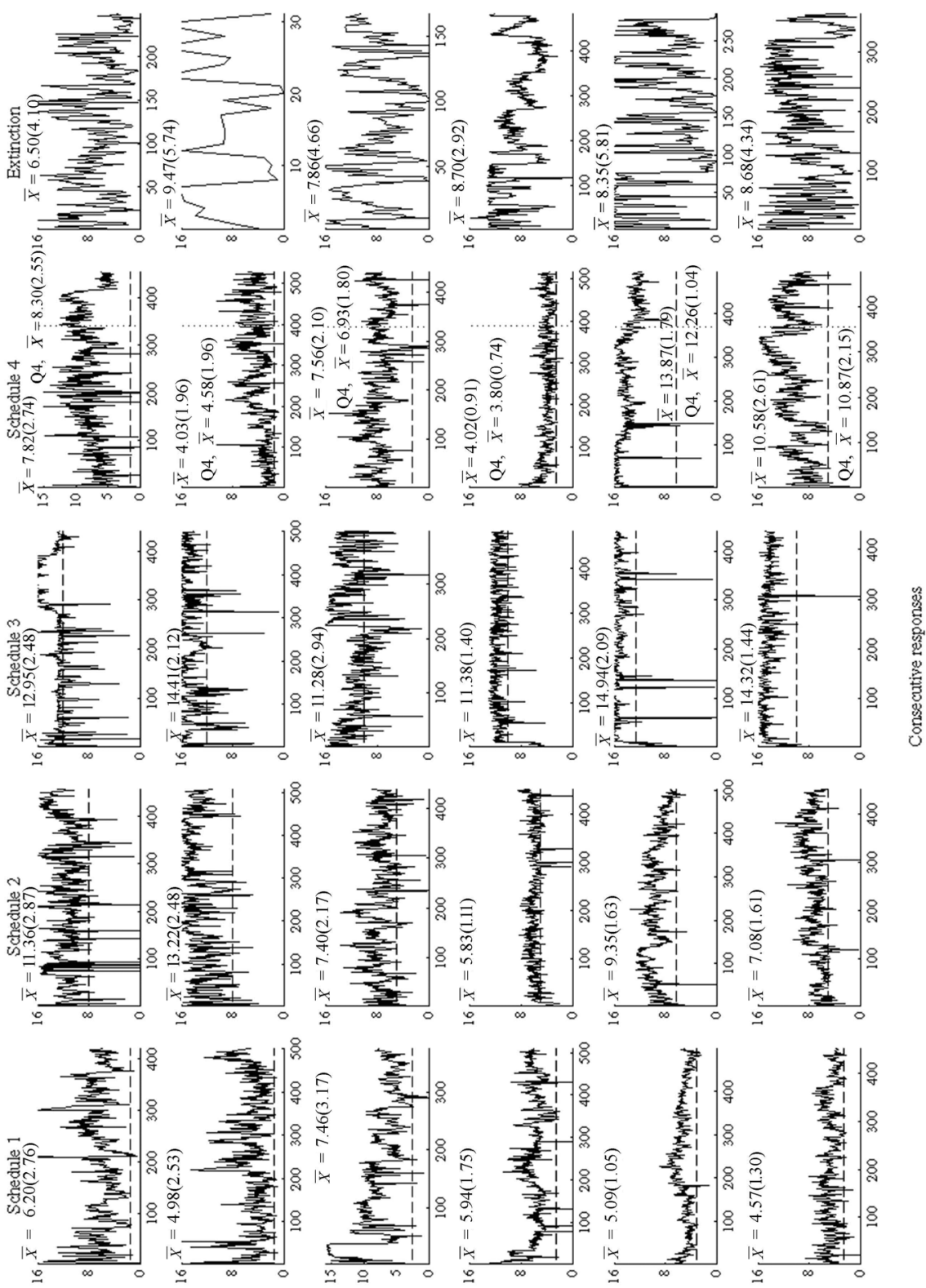

$\overrightarrow{\tilde{n}}$

ณ

$\stackrel{m}{m}$

若

$\stackrel{n}{m}$

$\ddot{\infty}$

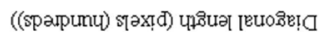

Figure 7. Consecutive diagonal lengths during each reinforcement schedule and extinction for each participant in Phase 1 of Experiment 3. IRT means $(\bar{X})$ are given on each graph, with the standard deviations $(S D)$ given in parentheses. For Schedule 4, both whole session and Q4 means and standard deviations are shown. 

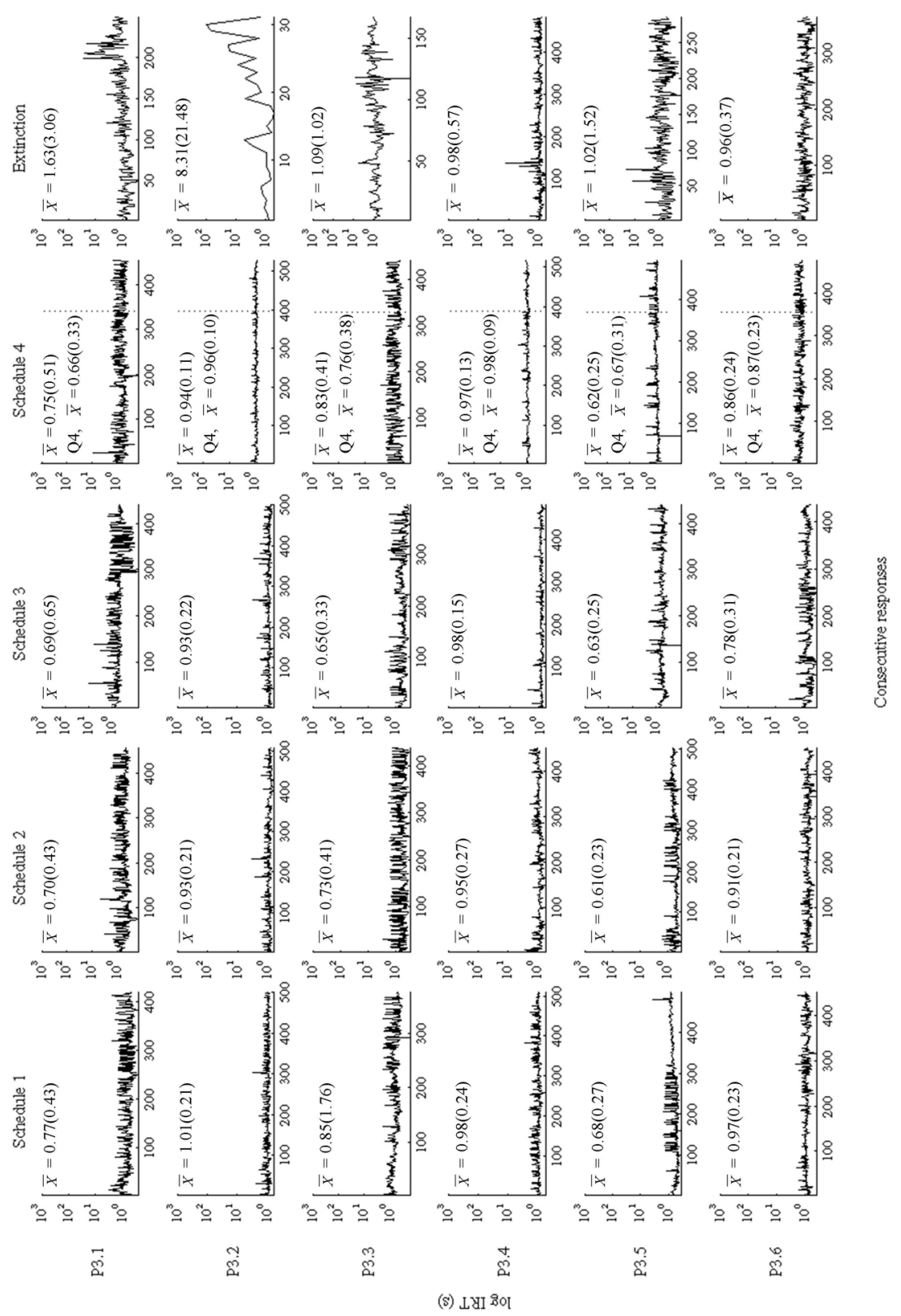

Figure 8. Consecutive IRTs during each reinforcement schedule and extinction for each participant in Phase 1 of Experiment 3. IRT means $(\bar{X})$ are given on each graph, with the standard deviations $(S D)$ given in parentheses. For Schedule 4, both whole session and Q4 means and standard deviations are shown. 
Schedule 1

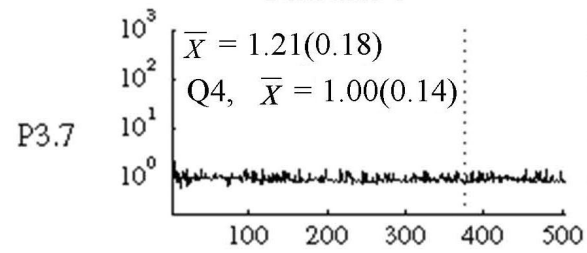

Extinction
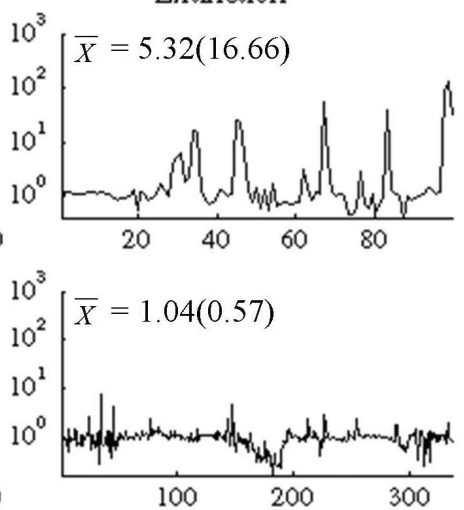
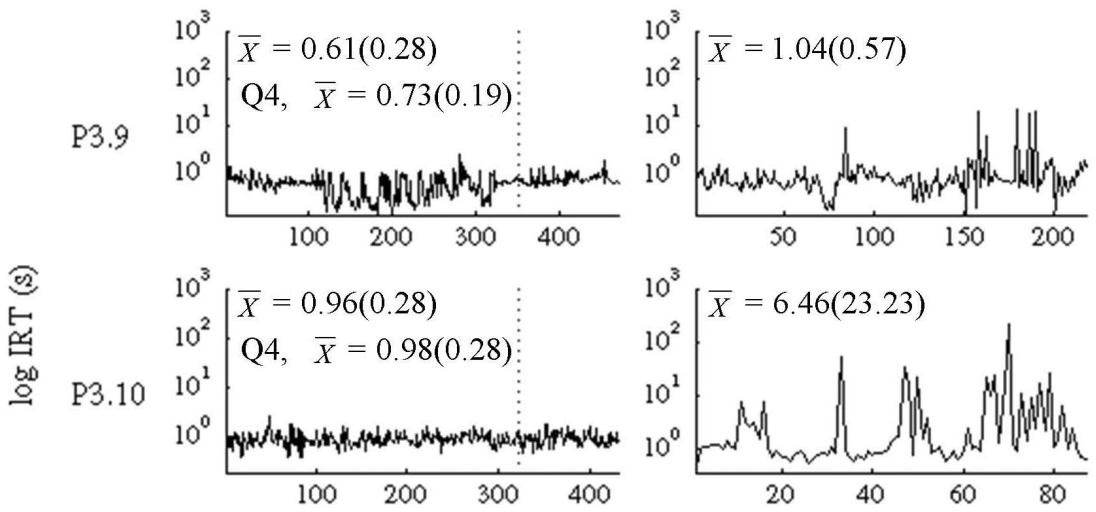

P3.8

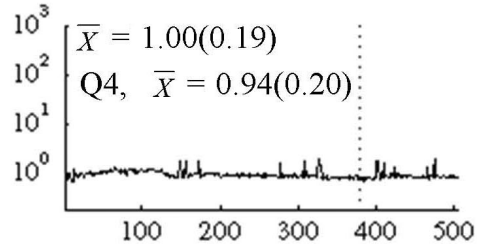

P3.11 $\begin{aligned} & 10^{1} \\ & 10^{2} \\ & 10^{\circ}\end{aligned}\left[\begin{array}{l}\bar{X}=0.74(0.41) \\ \mathrm{Q} 4, \bar{X}=0.54(0.36) \\ \vdots \\ 100 \quad 200 \quad 300 \quad 400\end{array}\right.$
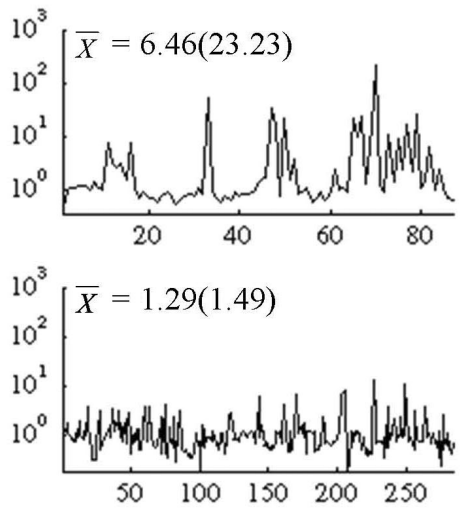

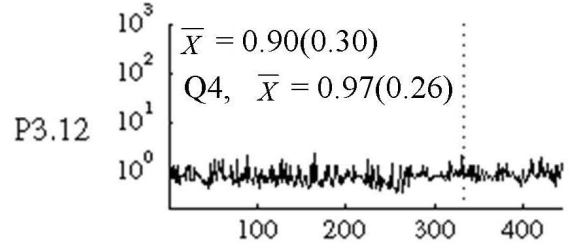

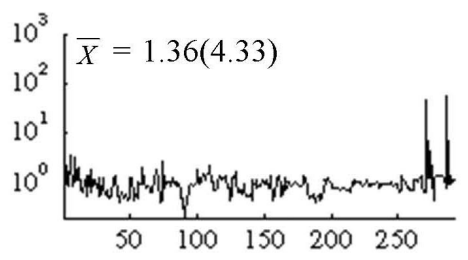

Consecutive responses

Figure 9. Consecutive diagonal lengths during the reinforcement schedule and extinction for each participant in Phase 2 of Experiment 3. Whole session and Q4 IRT means $(\bar{X})$ are given on each graph, with the standard deviations $(S D)$ given in parentheses. 
Schedule 1

$\bar{X}=5.54(1.93)$

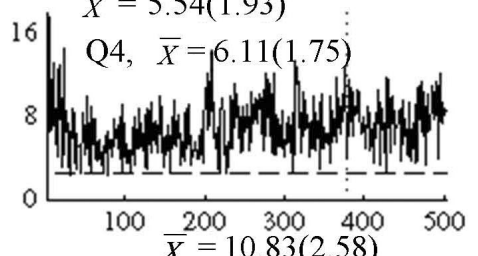

P3.7

P3.8

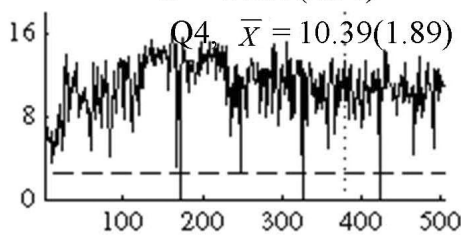
$\bar{X}=7.55(1.59)$

${ }^{16} \mid \mathrm{Q} 4, \bar{X}=8.87(1.34)$

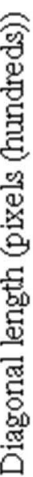

P3.11

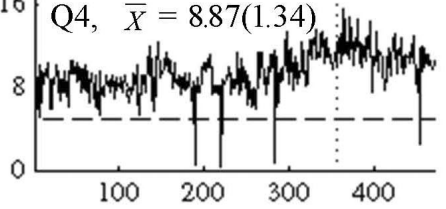
$\bar{X}=8.21(2.47)$

16 Q4, $\bar{X}=8.99(2.76)$

P3.10

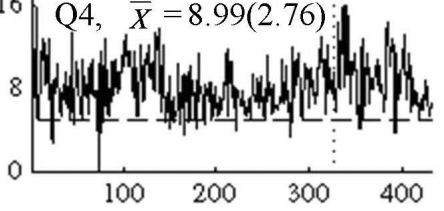
$\bar{X}=12.39(2.48)$

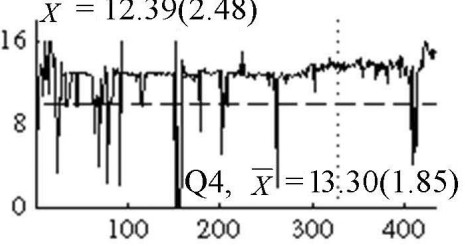
6. $\bar{X}=14.17(3.36)$

P3.12

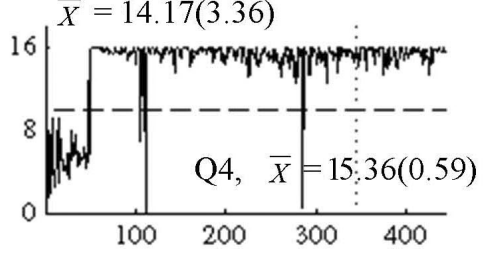

Extinction
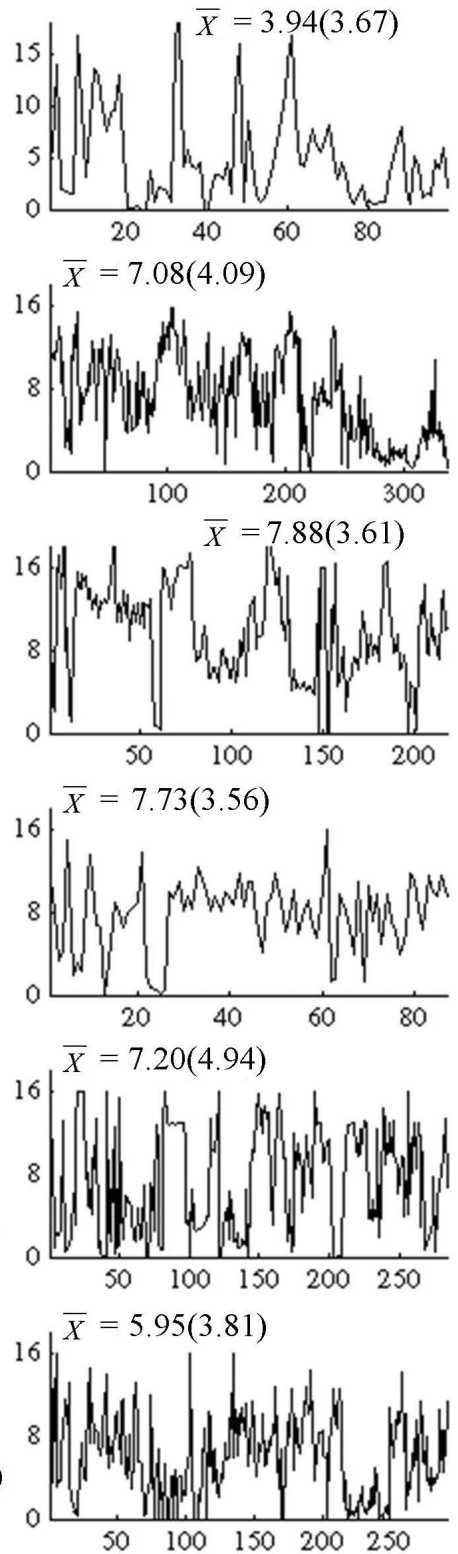

Consecutive responses

Figure 10. Consecutive IRTs during the reinforcement schedule and extinction for each participant in Phase 2 of Experiment 3. Whole session and Q4 IRT means $(\bar{X})$ are given on each graph, with the standard deviations $(S D)$ given in parentheses. 


\section{Discussion}

All participants showed greater variability in the contingent dimension (diagonal length) during extinction than during Q4 or the preceding schedules. This was similar to the finding for the contingent dimension (IRT) in Experiments 1 and 2, but the increase was not as great as it was in those two experiments. The increases in variability during extinction support the results of Morgan and Lee (1996). However, unlike these earlier findings with IRT, there was similar diagonal length variability during extinction in Phase 1 and 2. In terms of the noncontingent dimension (IRT), the results were similar to Experiment 2 (for diagonal length) with greater variability during extinction than during Q4, and similar variability in Phases 1 and 2.

One possible explanation for the lack of difference in variability of the contingent dimension between Phase 1 and 2 is the different constraints on the contingent dimensions of the response across experiments. Diagonal length was constrained by the size of the screen, with a maximum possible diagonal length of 1598.6 pixels, which was obtained by all of the participants during extinction. The uniformity of this as the maximum for each extinction condition suggests that the screen size acted as a ceiling on diagonal length and, had the experiment used a larger screen, greater diagonal length variability may have occurred. In contrast, IRT variability was only constrained by the length of the session (600 s). It was easily possible for the participants in Experiment 3 to have maximum values equal to the maximum possible diagonal length while still gaining reinforcement and meeting the stability criterion for each schedule, as there is very little difference in the length of time required to draw different size rectangles. However, DRL schedules, particularly long ones, require pauses in responding. As such, obtaining reinforcement for drawing rectangles is much easier than obtaining reinforcement under the DRL schedules. In the previous experiments, obtaining the maximum possible IRT would have required a participant to respond twice, once at each end of the session. This would not have been conducive to obtaining maximum reinforcement and would not have met the stability criterion for the session. This may also account for why the contingent dimension of the responses in the first two experiments (IRT) fell much closer to the reinforcement criterion and so was much less variable than the contingent dimension during the reinforcement conditions in Experiment 3.

Another possible explanation is that the changing criterion experienced by the participants in Phase 1 of each study was more salient in Experiments 1 and 2 than in Experiment 3. The IRTs recorded for the participants in both phases of Experiments 1 and 2 (once the criterion was met for the first time) tended to fall on or near the reinforcement criterion for that session. In addition, IRT decreased when the criterion decreased in the final schedule. Thus, in Phase 1 of Experiments 1 and 2, varying IRT was effectively reinforced across the different sessions, whereas in Phase 2, with only one schedule, there was no such history. A number of studies have demonstrated that variability in behavior can be an operant and can be changed by reinforcement (see Neuringer, 2004, for a review). It is possible that the different reinforcement histories could have contributed to the differences in the results from Phase 1 and 2. In Experiment 3, while the participants in Phase 1 experienced four different reinforcement schedules, they tended to produce diagonal lengths 
that were well above the criterion and that did not change significantly with changes in the criterion. Additionally, when the criterion was lowered during the final reinforcement schedule, diagonal length remained high and was similar to that in Phase 2. It could be argued then that the greater IRT variability in Phase 1 (compared to Phase 2) of Experiments 1 and 2 was a result of the participants in Phase 1 having experience of reinforcement for varying IRT. The lack of difference in the results from Phases 1 and 2 in Experiment 3 could be a result of the similarity in the reinforcement history of diagonal length.

The contingency during Experiment 3 was not time based, and this allowed much higher rates of responding than in Experiment 2. A very high proportion of responses in both phases of Experiment 3 was reinforced, with over $88 \%$ resulting in a reinforcer over all of the reinforcement schedules. These rates of reinforcement were much higher than for those in both phases of Experiments 1 and 2. Additionally, there was a much greater difference between the proportion of total responses that were reinforced in Phases 1 and 2 of the earlier experiments than there was in Experiment 3. In Experiments 1 and 2, a much higher proportion of responses was reinforced in Phase 1 than in Phase 2. It has been shown that different rates of reinforcement result in differences in the degree to which behavior changes, relative to its preextinction level, in extinction. Nevin, Mandell, and Atak (1983) reported that the higher the rate of reinforcement associated with a response, the greater its resistance to change when disrupted by processes such as extinction; that is, it has greater behavioral momentum. It is possible then that the different rates of reinforcement over the phases in Experiments 1 and 2 contributed to the differences in IRT variability during extinction between the phases. That is, the difference in reinforcement history may be the different proportions of responses that were reinforced, rather than the different number of schedules experienced. To study the resistance to change further would require a longer extinction condition than used here.

There was a further difference between Experiments 1 and 2 and Experiment 3. The instructions given to each set of participants were the same; however, reinforcement was delivered under a different contingency in Experiment 3. In Experiment 3, the participants were instructed to respond as often as they liked. Participants in the first two experiments were required to wait between responses, and this instruction was contrary to the behavior needed to gain the maximum possible reinforcers in a session. In Experiment 3 this was not the case, and responding as often as they liked would give a greater overall reinforcement rate. It is not clear to what degree this might have given rise to the different patterns of responding, but it is possible that this contributed to the development of the different reinforcement histories.

\section{General Discussion}

All three experiments replicated Morgan and Lee's (1996) finding that extinction results in greater variability in the contingent dimension of the response. This finding applied not only to responses during the last quarter of the final reinforcement session, as in the Morgan and Lee study, but also when data from the whole of the reinforcement schedules were considered. Experiment 2 generalized this result across tasks. Experiment 3 found similar results on a different contingent dimension. 
Unlike Morgan and Lee (1996), the findings of Experiments 1 and 2 suggested that reinforcement history did affect variability of behavior during extinction. In Experiment 3 there appeared to be no effect of the number of contingency changes on variability in diagonal length (the contingent dimension). One possible explanation for this difference is the different histories of reinforcement for varying the contingent dimension. Another possibility is that having a contingency based on diagonal length (an easier task) resulted in participants making a higher proportion of reinforced responses prior to extinction than they did with the DRL schedules. Thus, although participants in the two phases of the experiment experienced different numbers of schedule changes, they all experienced high numbers of reinforced responses. In this respect, they had similar histories of reinforcement in both phases and so had similar behavior in extinction.

The response requirement in Experiments 2 and 3 (drawing rectangles) is a limitation of this study. The goal was to find a response that could vary in more than one measurable dimension. Large and small diagonals took about the same time to produce, so varying the diagonal length criterion for reinforcement did not alter the duration of the response. As a result, in Experiment 3 the participants could draw the maximum diagonal length consistently and still maintain close to maximum reinforcement rates. Thus, there was no real contingency on reducing length when the criterion was reduced. The fact that the majority of participants in Experiment 3 regularly drew diagonals with the maximum possible diagonal length suggests that, had there been the opportunity, much more variability would have been observed on this dimension.

The present study provides the first detailed examination of human performance on the noncontingent dimension of a response when the contingent dimension is subject to extinction. This demonstrates that extinction generates variability in both the contingent and noncontingent dimensions of a response. This has important implications for the development of new behavior, as variability across a range of response dimensions allows, and aids, the selection of a new form of the response to replace the original but now ineffective behavior.

\section{References}

ANTONITIS, J. J. (1951). Response variability in the white rat during conditioning, extinction, and reconditioning. Journal of Experimental Psychology, 42, 273-281.

DEWITTE, S., \& VERGUTS, T. (1999). Behavioral variation: A neglected aspect in selectionist thinking. Behavior and Philosophy, 27, 127-145.

ECKERMAN, D. A., \& LANSON, R. N. (1969). Variability of response location for pigeons responding under continuous reinforcement, intermittent reinforcement, and extinction. Journal of the Experimental Analysis of Behavior, 12(1), 73-80.

HERRNSTEIN, R. J. (1961). Stereotypy and intermittent reinforcement. Science, 133, 2067-2069.

LACHTER, G. D., \& COREY, J. R. (1982). Variability of the duration of an operant. Behavior Analysis Letters, 2(2), 97-102.

MAES, J. H. R. (2003). Response stability and variability induced in humans by different feedback contingencies. Learning \& Behavior, 31(4), 332-348. 
MORGAN, D. L., \& LEE, K. (1996). Extinction-induced response variability in humans. The Psychological Record, 46(1), 145-159.

NEURINGER, A. (2002). Operant variability: Evidence, functions, and theory. Psychonomic Bulletin \& Review, 9(4), 672-705.

NEURINGER, A. (2004). Reinforced variability in animals and people: Implications for adaptive action. American Psychologist, 59(9), 891-906.

NEURINGER, A., DEISS, C., \& OLSEN, G. (2000). Reinforced variability and operant learning. Journal of Experimental Psychology, 26(1), 98-111.

NEURINGER, A., KORNELL, N., \& OLUFS, M. (2001). Stability and variability in extinction. Journal of Experimental Psychology, 27(1), 79-94.

NEVIN, J. A., MANDELL, C., \& ATAK, J. R. (1983). The analysis of behavioral momentum. Journal of the Experimental Analysis of Behavior, 39(1), 49-59.

ROSS, C., \& NEURINGER, A. (2002). Reinforcement of variations and repetitions along three independent response dimensions. Behavioral Processes. Special Issue: Proceedings of the meeting of the Society for the Quantitative Analyses of Behavior (SQAB 2001), 57(2-3), 199-209.

STOKES, P. D. (1995). Learned variability. Animal Learning \& Behavior, 23(2), 164-176. 
